\title{
High density single-channel seismic profiles obtained during KY05-06 cruise in the Sagami Bay, Japan
}

\author{
Masataka Kinoshita ${ }^{1}$, Mikiya Yamashita ${ }^{1}$, Tadashi Okano², Satoshi Shimizu ${ }^{3}$, Yui Hashimoto ${ }^{3}$, \\ Takafumi Kasaya ${ }^{1}$ and KY05-06 Leg2 Shipboard Scientific Party*
}

* Hideaki Machiyama ${ }^{1 \dagger}$, Kyohiko Mitsuzawa $^{4}$ and Keizo Sayanagi ${ }^{5}$

\begin{abstract}
In May, 2005, intensive single channel seismic surveys have been successfully completed in Sagami Bay area, central Japan, during the KY05-06 cruise using R/V KAIYO of JAMSTEC (Japan Agency for Marine-Earth Science and Technology). We obtained 22 SCS profiles in the overall Sagami Bay area, and 37 shallow and deep profiles around the Hatsushima cold seep communities in the western Sagami Bay. Very clear acoustic images were captured down to 1 sec. (TWT) after some editing, band-pass filtering and time migration. We present here the seismic profiles obtained during the KY05-06 cruise.
\end{abstract}

Keywords: Sagami-bay, Single Channel Seismic Survey, Hatsushima cold seep community, Cabled observatory

\section{Introduction}

The Sagami Bay, central Japan, is known to be in the most complicated tectonics in the world. Subduction of Philippine Sea Plate beneath mainland Honshu is significantly obstructed by the collision of Izu Peninsula. The volcanic front of Izu-Bonin Arc extends northward from Izu-Oshima through Higashi-Izu monogenetic volcanoes. Active cold seep biological communities are distributed along the escarpment of the eastern rim of the Izu Peninsula. Extensive earthquake swarms repeatedly occur in the eastern Izu region (Okada et al., 2000).

Intensive geological, geophysical, geochemical and biological surveys have been carried out in this area. One of the most basic items is seismic reflection survey (single-channel or multi-channel), which has been conducted in Japan (e.g. Iwabuchi et al., 1990; Kato et al., 1993). However, most records were in an analog format and are difficult to investigate in detail. Besides, intervals between survey lines are too sparse to see any hydrologeological features in detail beneath the Hatsushima cold seep site.

We conducted high-density single channel seismic (SCS) survey in overall Sagami Bay at every $4 \mathrm{~km}$ interval (Fig. 1), and around the Hatsushima cold seep site at 0.1-0.2 miles interval (Fig. 2). We here briefly report

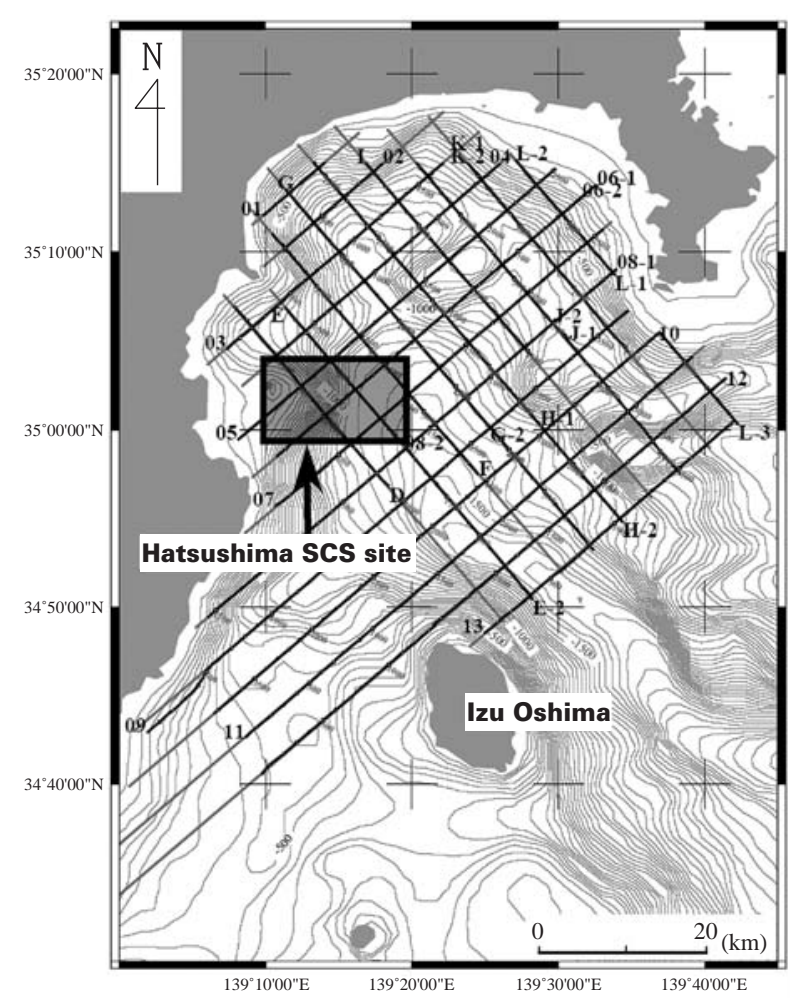

Figure 1: Index map of Sagami Bay with SCS survey tracks. Square indicates the area for intensive survey off Hatsushima.

\footnotetext{
1 IFREE, Japan Agency for Marine-Earth Science and Technology

2 CDEX, Japan Agency for Marine-Earth Science and Technology

3 Nippon Marine Enterprise, Co. Ltd.

4 MARITEC, Japan Agency for Marine-Earth Science and Technology

5 Tokai University

$\dagger$ Now at Kochi Institute for Core Sample Research, Japan Agency for Marine-Earth Science and Technology
} 


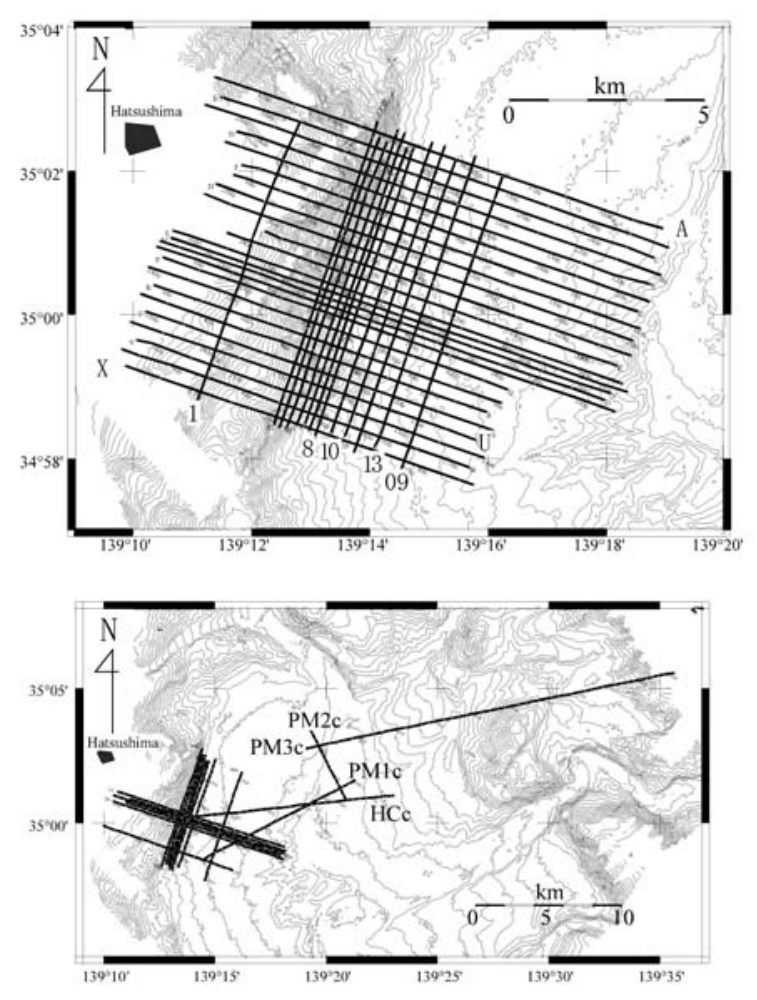

Figure 2: (Upper panel) SCS survey track in the Hatsushima site (the area shown as a square in Fig. 1). (Lower panel) Other SCS survey track around the Hatsushima site.

the result of KY05-06 cruise, providing preliminary profiles obtained during this cruise.

\section{Data acquisition}

\subsection{Overall Sagami Bay Survey}

SCS survey was carried out from April 25, 2005 to May 5, 2005, using JAMSTEC R/V KAIYO with a total length of approx. $852 \mathrm{~km}$ (Fig. 1). The GI gun system was used, with the line spacing $4 \mathrm{~km}$.

Survey lines were planed based on the following considerations;

(1) Survey lines would cover Sagami-bay area extensively.

(2) Direction of survey lines would be decided based on the structural trend in this area.

(3) Survey lines will be prepared to be convenient for the construction of structural map, though resolution in the deeper part is limited because of SCS data collection.

(4) The southern part of Sagami Bay has a very heavy, ship traffic lane for Tokyo Bay, so survey lines were adjusted from the points of safe ship traffic.

Survey line information is summarized in Table 1.

\subsection{Hatsushima Intensive Survey}

SCS survey was carried out from May 5 to May 10, 2005, onboard JAMSTEC R/V KAIYO with a total

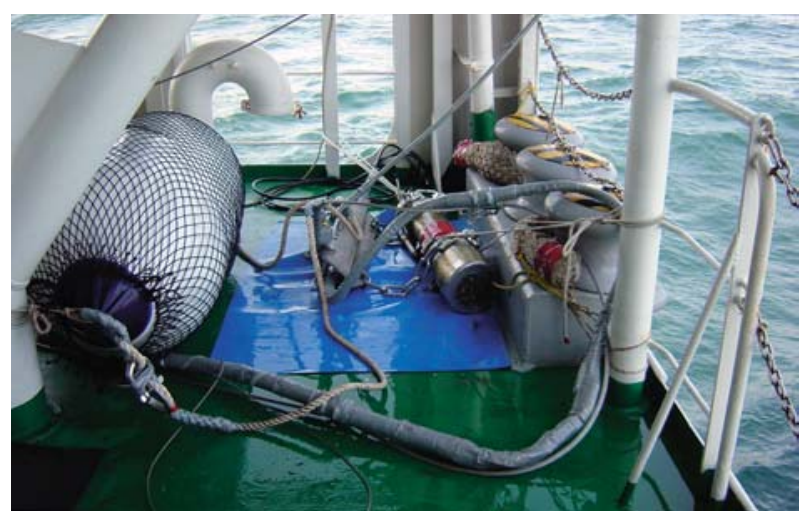

Figure 3: Photographs of the GI gun system

length of approx. $300 \mathrm{~km}$ (Fig. 2). A dense grid survey was carried out, centered at the Hatsushima cold seep site, with the line spacing 0.1-0.2 miles. In order to obtain shallow structure as well as deeper imagery, we first deployed GI gun system (105 cuin, steaming at 5 knots, 8 sec. shot interval, $50 \mathrm{~m}$ streamer length). After that CDEX's cluster gun system was replaced to cover the shallower images at some lines (20 cuin, steaming at 3 knots, $3 \mathrm{sec}$. shot interval, $20 \mathrm{~m}$ streamer length) (Fig. 3 ). Table 2 shows this survey line information.

\section{SCS system and processing summary 3.1 GI gun system}

The GI gun system is effective to obtain acoustic images down to $1 \mathrm{sec}$ (TWT).

GI gun

Total volume: 355 cuin (Generator 250 cuin/ Injector 105 cuin)

Towing depth: $3 \mathrm{~m}$

Source pressure: 2,000 psi (136 atm.)

Pop interval: 8 seconds

\section{Streamer}

Seamap streamer cable was used for the first line SG05SCS-05. Since noisy traces appeared for this cable, we replaced it with SIG16 streamer cable, which worked fine throughout the rest of the lines.

Seamap streamer specifications:

Streamer length: $10 \mathrm{~m}$

No. of Hydrophones: 20

Hydrophone interval: $0.5 \mathrm{~m}$

Type of Hydrophone: SQ5 Sensor Technology

Frequency: $1 \mathrm{~Hz}-6,000 \mathrm{~Hz}+/-0.5 \mathrm{~dB}$

Preamp: $22.2 \mathrm{~dB}$

SIG16 streamer specifications:

Streamer length: $50 \mathrm{~m}$

No. of Hydrophones: 48

Hydrophone interval: $1 \mathrm{~m}$

Type of Hydrophone: SIG

Frequency: $10 \mathrm{~Hz}-1,000 \mathrm{~Hz}+/-1 \mathrm{~dB}$ 
Table 1: SCS line information for the overall Sagami Bay survey

\begin{tabular}{|c|c|c|c|c|c|}
\hline Line Name & Starting point & Ending point & Shooting Direction & SP number & Line length $(\mathrm{km})$ \\
\hline SG-05SCS -01 & $\begin{array}{r}35^{\circ} 12^{\prime} 02.17^{\prime \prime} \mathrm{N} \\
139^{\circ} 09^{\prime} 50.03 \text { " E }\end{array}$ & $\begin{array}{r}35^{\circ} 14^{\prime} 57.88^{\prime \prime} \mathrm{N} \\
139^{\circ} 13,59.51 ” \mathrm{E}\end{array}$ & $\mathrm{NE}$ & $2-495$ & 8.15 \\
\hline-02 & $\begin{array}{r}35^{\circ} 15^{\prime} 01.83^{\prime \prime} \mathrm{N} \\
139^{\circ} 18^{\prime} 06.30 ” \mathrm{E}\end{array}$ & $\begin{array}{r}35^{\circ} 10^{\prime} 12.06 ” \mathrm{~N} \\
139^{\circ} 11,15.73 ” \mathrm{E}\end{array}$ & SW & $2-821$ & 13.53 \\
\hline-03 & $\begin{array}{r}35^{\circ} 04^{\prime} 32.88^{\prime \prime} \mathrm{N} \\
139^{\circ} 07^{\prime} 27.24 ” \mathrm{E}\end{array}$ & $\begin{array}{r}35^{\circ} 14^{\prime} 54.44 " \mathrm{~N} \\
139^{\circ} 22^{\prime} 08.16^{\prime \prime} \mathrm{E}\end{array}$ & $\mathrm{NE}$ & $20-1695$ & 27.65 \\
\hline-04 & $\begin{array}{r}35^{\circ} 15^{\prime} 01.50^{\prime \prime} \mathrm{N} \\
139^{\circ} 26^{\prime} 06.15^{\prime \prime} \mathrm{E}\end{array}$ & $\begin{array}{r}35^{\circ} 04^{\prime} 01.30^{\prime \prime} \mathrm{N} \\
139^{\circ} 10^{\prime} 31.49^{\prime \prime} \mathrm{E}\end{array}$ & SW & $2-1821$ & 30.03 \\
\hline-05 & $\begin{array}{r}34^{\circ} 59^{\prime} 25.11^{\prime \prime} \mathrm{N} \\
139^{\circ} 08^{\prime} 06.68^{\prime \prime} \mathrm{E}\end{array}$ & $\begin{array}{r}35^{\circ} 14^{\prime} 38.15^{\prime \prime} \mathrm{N} \\
139^{\circ} 29^{\prime} 44.38^{\prime \prime} \mathrm{E}\end{array}$ & $\mathrm{NE}$ & $13-2517$ & 41.33 \\
\hline$-06-1$ & $\begin{array}{r}35^{\circ} 13^{\prime} 48.34 ” \mathrm{~N} \\
139^{\circ} 32^{\prime} 40.32^{\prime \prime} \mathrm{E}\end{array}$ & $\begin{array}{r}35^{\circ} 11^{\prime} 59.33 ” \mathrm{~N} \\
139^{\circ} 29^{\prime} 53.31 ” \mathrm{E}\end{array}$ & SW & $7-321$ & 5.20 \\
\hline$-06-2$ & $\begin{array}{r}35^{\circ} 13^{\prime} 02.63 ” \mathrm{~N} \\
139^{\circ} 31,33.14 ” \mathrm{E}\end{array}$ & $\begin{array}{r}34^{\circ} 58^{\prime} 32.06 ” \mathrm{~N} \\
139^{\circ} 10^{\prime} 49.53^{\prime \prime} \mathrm{E}\end{array}$ & SW & $19-2375$ & 38.89 \\
\hline-07 & $\begin{array}{r}34^{\circ} 55^{\prime} 46.30^{\prime \prime} \mathrm{N} \\
139^{\circ} 10^{\prime} 49.84 " \mathrm{E}\end{array}$ & $\begin{array}{r}35^{\circ} 11^{\prime} 13.05 " \mathrm{~N} \\
139^{\circ} 33,00.89^{\prime \prime} \mathrm{E}\end{array}$ & $\mathrm{NE}$ & $26-2568$ & 41.96 \\
\hline$-08-1$ & $\begin{array}{r}35^{\circ} 09^{\prime} 05.45^{\prime \prime} \mathrm{N} \\
139^{\circ} 34^{\prime} 03.37^{\prime \prime} \mathrm{E}\end{array}$ & $\begin{array}{r}34^{\circ} 58^{\prime} 26.22 ” \mathrm{~N} \\
139^{\circ} 18^{\prime}, 51.21^{\prime \prime} \mathrm{E}\end{array}$ & SW & $2-1783$ & 29.40 \\
\hline$-08-2$ & $\begin{array}{r}34^{\circ} 58^{\prime} 42.30^{\prime \prime} \mathrm{N} \\
139^{\circ} 19^{\prime} 16.57^{\prime \prime} \mathrm{E}\end{array}$ & $\begin{array}{r}34^{\circ} 49^{\prime} 27.16^{\prime \prime} \mathrm{N} \\
139^{\circ} 06^{\prime} 07.57^{\prime \prime} \mathrm{E}\end{array}$ & SW & $37-1568$ & 25.28 \\
\hline-09 & $\begin{array}{r}34^{\circ} 46^{\prime} 32.87^{\prime \prime} \mathrm{N} \\
139^{\circ} 06^{\prime} 05.11^{\prime \prime} \mathrm{E}\end{array}$ & $\begin{array}{r}35^{\circ} 06^{\prime} 43.10^{\prime \prime} \mathrm{N} \\
139^{\circ} 34^{\prime} 48.29^{\prime \prime} \mathrm{E}\end{array}$ & $\mathrm{NE}$ & $523-3736$ & 53.03 \\
\hline-10 & $\begin{aligned} & 35^{\circ} 05^{\prime} 28.67^{\prime \prime} \mathrm{N} \\
& 139^{\circ} 36^{\prime} 53.95^{\prime \prime} \mathrm{E}\end{aligned}$ & $\begin{array}{r}34^{\circ} 44^{\prime} 02.79 ” \mathrm{~N} \\
139^{\circ} 06^{\prime} 32.91 ” \mathrm{E}\end{array}$ & SW & $2-3832$ & 63.21 \\
\hline-11 & $\begin{array}{r}34^{\circ} 42^{\prime} 33.73^{\prime \prime} \mathrm{N} \\
139^{\circ} 08^{\prime} 33.97^{\prime \prime} \mathrm{E}\end{array}$ & $\begin{array}{r}35^{\circ} 04^{\prime} 03.83 ” \mathrm{~N} \\
139^{\circ} 39^{\prime} 06.10^{\prime \prime} \mathrm{E}\end{array}$ & $\mathrm{NE}$ & $2-3445$ & 56.83 \\
\hline$-12-1$ & $\begin{array}{r}35^{\circ} 02^{\prime} 53.65^{\prime \prime} \mathrm{N} \\
139^{\circ} 41^{\prime} 27.06 ” \mathrm{E}\end{array}$ & $\begin{array}{r}34^{\circ} 49^{\prime} 00.50^{\prime \prime} \mathrm{N} \\
139^{\circ} 21^{\prime} 30.12^{\prime \prime} \mathrm{E}\end{array}$ & SW & $10-2477$ & 40.72 \\
\hline$-12-2$ & $\begin{array}{r}34^{\circ} 40^{\prime} 42.56^{\prime \prime} \mathrm{N} \\
139^{\circ} 09^{\prime} 49.87^{\prime \prime} \mathrm{E}\end{array}$ & $\begin{array}{r}34^{\circ} 48^{\prime} 57.44^{\prime \prime} \mathrm{N} \\
139^{\circ} 21^{\prime} 35.38^{\prime \prime} \mathrm{E}\end{array}$ & $\mathrm{NE}$ & $50-1359$ & 21.62 \\
\hline-13 & $\begin{aligned} 34^{\circ} 48^{\prime} & 35.36^{\prime \prime} \mathrm{N} \\
139^{\circ} 25^{\prime} & 03.14 ” \mathrm{E}\end{aligned}$ & $\begin{array}{r}35^{\circ} 00^{\prime} 24.16 " \mathrm{~N} \\
139^{\circ} 41^{\prime} 52.66 ” \mathrm{E}\end{array}$ & $\mathrm{NE}$ & $24-1921$ & 31.32 \\
\hline$-\mathrm{D}$ & $\begin{array}{r}34^{\circ} 56^{\prime} 54.69^{\prime \prime} \mathrm{N} \\
139^{\circ} 18^{\prime} 14.35^{\prime \prime} \mathrm{E}\end{array}$ & $\begin{array}{r}35^{\circ} 06^{\prime} 53.82 ” \mathrm{~N} \\
139^{\circ} 07,54.66^{\prime \prime} \mathrm{E}\end{array}$ & NW & $27-1408$ & 22.80 \\
\hline$-\mathrm{E}-1$ & $\begin{array}{r}35^{\circ} 06^{\prime} 56.41 ” \mathrm{~N} \\
139^{\circ} 11^{\prime} 16.44 ” \mathrm{E}\end{array}$ & $\begin{array}{r}34^{\circ} 56^{\prime} 00.07^{\prime \prime} \mathrm{N} \\
139^{\circ} 22^{\prime} 39.18^{\prime \prime} \mathrm{E}\end{array}$ & SE & $2-1544$ & 25.46 \\
\hline$-\mathrm{E}-2$ & $\begin{array}{r}34^{\circ} 50^{\prime} 26.66^{\prime \prime} \mathrm{N} \\
139^{\circ} 28^{\prime} 13.87^{\prime \prime} \mathrm{E}\end{array}$ & $\begin{array}{r}34^{\circ} 57^{\prime} 14.92 ” \mathrm{~N} \\
139^{\circ} 21^{\prime} 22.08^{\prime \prime} \mathrm{E}\end{array}$ & NW & $9-958$ & 15.68 \\
\hline$-\mathrm{F}$ & $\begin{array}{r}34^{\circ} 57^{\prime} 23.94 ” \mathrm{~N} \\
139^{\circ} 24^{\prime} 36.24 ” \mathrm{E}\end{array}$ & $\begin{array}{r}35^{\circ} 10^{\prime} 58.14 " \mathrm{~N} \\
139^{\circ} 10^{\prime} 29.69^{\prime \prime} \mathrm{E}\end{array}$ & NW & $6-1897$ & 31.22 \\
\hline$-\mathrm{G}-1$ & $\begin{array}{r}35^{\circ} 13^{\prime} 29.36^{\prime \prime} \mathrm{N} \\
139^{\circ} 11^{\prime} 24.69^{\prime \prime} \mathrm{E}\end{array}$ & $\begin{array}{r}34^{\circ} 58^{\prime} 53.63^{\prime \prime} \mathrm{N} \\
139^{\circ} 26^{\prime} 38.70^{\prime \prime} \mathrm{E}\end{array}$ & SE & $11-2009$ & 32.98 \\
\hline$-\mathrm{G}-2$ & 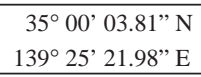 & 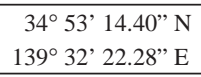 & SE & $18-958$ & 15.53 \\
\hline$-\mathrm{H}-1$ & $\begin{array}{r}35^{\circ} 00^{\prime} 14.47^{\prime \prime} \mathrm{N} \\
139^{\circ} 28^{\prime} 44.83^{\prime \prime} \mathrm{E}\end{array}$ & $\begin{array}{r}35^{\circ} 14^{\prime} 57.38^{\prime \prime} \mathrm{N} \\
139^{\circ} 13,21.80 ” \mathrm{E} \\
\end{array}$ & NW & $11-2108$ & 34.62 \\
\hline$-\mathrm{H}-2$ & $\begin{array}{r}34^{\circ} 54^{\prime} 43.30^{\prime \prime} \mathrm{N} \\
139^{\circ} 34^{\prime} 28.12 ” \mathrm{E}\end{array}$ & $\begin{array}{r}35^{\circ} 01^{\prime} 36.20^{\prime \prime} \mathrm{N} \\
139^{\circ} 27,20.55^{\prime \prime} \mathrm{E}\end{array}$ & NW & $2-1004$ & 16.55 \\
\hline$-\mathrm{I}$ & $\begin{array}{r}35^{\circ} 15^{\prime} 01.14 " \mathrm{~N} \\
139^{\circ} 16^{\prime} 47.11^{\prime \prime} \mathrm{E}\end{array}$ & 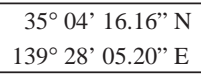 & SE & $6-1531$ & 25.18 \\
\hline$-\mathrm{J}-1$ & $\begin{array}{r}35^{\circ} 05^{\prime} 02.24^{\prime \prime} \mathrm{N} \\
139^{\circ} 30^{\prime} 46.01^{\prime \prime} \mathrm{E}\end{array}$ & $\begin{array}{r}35^{\circ} 14^{\prime} 56.17 ” \mathrm{~N} \\
139^{\circ} 20^{\prime} 22.50^{\prime \prime} \mathrm{E}\end{array}$ & NW & $12-1433$ & 23.46 \\
\hline$-\mathrm{J}-2$ & $\begin{array}{r}35^{\circ} 06^{\prime} 03.96^{\prime \prime} \mathrm{N} \\
139^{\circ} 29^{\prime} 35.65^{\prime \prime} \mathrm{E}\end{array}$ & $\begin{array}{r}34^{\circ} 57^{\prime} 31.76 ” \mathrm{~N} \\
139^{\circ} 38^{\prime} 19.03 " \mathrm{E}\end{array}$ & SE & $2-1175$ & 19.37 \\
\hline$-\mathrm{K}-1$ & $\begin{array}{r}35^{\circ} 15^{\prime} 00.00^{\prime \prime} \mathrm{N} \\
139^{\circ} 23^{\prime} 48.16^{\prime \prime} \mathrm{E}\end{array}$ & $\begin{array}{r}35^{\circ} 11^{\prime} 26.10 ” \mathrm{~N} \\
139^{\circ} 27,26.66 ” \mathrm{E}\end{array}$ & SE & $8-529$ & 8.61 \\
\hline$-\mathrm{K}-2$ & $\begin{array}{r}35^{\circ} 15^{\prime} 03.12^{\prime \prime} \mathrm{N} \\
139^{\circ} 23^{\prime} 45.43^{\prime \prime} \mathrm{E}\end{array}$ & $\begin{array}{r}35^{\circ} 04^{\prime} 24.94 " \mathrm{~N} \\
139^{\circ} 34^{\prime} 41.25 " \mathrm{E}\end{array}$ & SE & $2-1538$ & 25.36 \\
\hline -L-1 & $\begin{array}{r}35^{\circ} 08^{\prime} 38.46^{\prime \prime} \mathrm{N} \\
139^{\circ} 33^{\prime} 55.85^{\prime \prime} \mathrm{E}\end{array}$ & $\begin{array}{r}35^{\circ} 11^{\prime} 42.46 ” \mathrm{~N} \\
139^{\circ} 30^{\prime} 41.30^{\prime \prime} \mathrm{E}\end{array}$ & NW & $3-443$ & 7.28 \\
\hline -L-2 & $\begin{array}{r}35^{\circ} 15^{\prime} 07.78^{\prime \prime} \mathrm{N} \\
139^{\circ} 27^{\prime} 06.81^{\prime \prime} \mathrm{E}\end{array}$ & $\begin{array}{r}35^{\circ} 11^{\prime} 40.66 " \mathrm{~N} \\
139^{\circ} 30^{\prime} 43.08 " \mathrm{E}\end{array}$ & SE & $15-504$ & 8.09 \\
\hline -L-3 & $\begin{array}{r}35^{\circ} 00^{\prime} 12.99^{\prime \prime} \mathrm{N} \\
139^{\circ} 42^{\prime} 21.43^{\prime \prime} \mathrm{E}\end{array}$ & $\begin{array}{r}35^{\circ} 05^{\prime} 27.66^{\prime \prime} \mathrm{N} \\
139^{\circ} 36,56.02^{\prime \prime} \mathrm{E}\end{array}$ & NW & $2-718$ & 11.83 \\
\hline & & & & Total & 852.17 \\
\hline
\end{tabular}

Line lengths in this table were derived from the calculation of number of shots multiplied by $16.5 \mathrm{~m}$ ( 8 seconds pop interval approximately corresponds $16.5 \mathrm{~m}$ when 4 knots cruising speed). 
Table 2: SCS line information for the intensive Hatsushima survey

\begin{tabular}{|c|c|c|c|c|c|}
\hline Line Name & Starting point & Ending point & Shooting Direction & SP number & Line length $(\mathrm{km})$ \\
\hline KY0506H-01 & $\begin{array}{r}35^{\circ} 02.6923^{\prime} \mathrm{N} \\
139^{\circ} 12.8275^{\prime} \mathrm{E}\end{array}$ & $\begin{array}{r}34^{\circ} 58.8048^{\prime} \mathrm{N} \\
139^{\circ} 11.0900^{\prime} \mathrm{E}\end{array}$ & SSW & $2-357$ & 4.22 \\
\hline KY0506H-02 & $\begin{array}{r}34^{\circ} 58.4643^{\prime} \mathrm{N} \\
139^{\circ} 12.3770^{\prime} \mathrm{E} \\
\end{array}$ & $\begin{array}{r}35^{\circ} 02.6886^{\prime} \mathrm{N} \\
139^{\circ} 14.1373, \mathrm{E} \\
\end{array}$ & NNE & $2-475$ & 7.82 \\
\hline KY0506H-03 & $\begin{array}{r}35^{\circ} 02.4785^{\prime} \mathrm{N} \\
139^{\circ} 14.1705^{\prime} \mathrm{E}\end{array}$ & $\begin{array}{r}34^{\circ} 58.4283^{\prime} \mathrm{N} \\
139^{\circ} 12.4696^{\prime} \mathrm{E} \\
\end{array}$ & SSW & $1323-1706$ & 6.34 \\
\hline KY0506H-04 & $\begin{array}{r}35^{\circ} 02.4161^{\prime} \mathrm{N} \\
139^{\circ} 14.2907^{\prime} \mathrm{E}\end{array}$ & $\begin{array}{r}34^{\circ} 58.3987^{\prime} \mathrm{N} \\
139^{\circ} 12.5883^{\prime} \mathrm{E} \\
\end{array}$ & SSW & $479-853$ & 6.19 \\
\hline KY0506H-05 & $\begin{array}{r}34^{\circ} 58.4514^{\prime} \mathrm{N} \\
139^{\circ} 12.7613^{\prime} \mathrm{E} \\
\end{array}$ & $\begin{array}{r}35^{\circ} 02.5788^{\prime} \mathrm{N} \\
139^{\circ} 14.47411^{\prime} \mathrm{E} \\
\end{array}$ & NNE & $1710-2156$ & 7.38 \\
\hline KY0506H-06 & $\begin{array}{r}34^{\circ} 58.4785^{\prime} \mathrm{N} \\
139^{\circ} 12.9088^{\prime} \mathrm{E}\end{array}$ & $\begin{array}{r}35^{\circ} 02.5365^{\prime} \mathrm{N} \\
139^{\circ} 14.5949^{\prime} \mathrm{E}\end{array}$ & NNE & $857-1319$ & 7.64 \\
\hline KY0506H-07 & $\begin{array}{r}35^{\circ} 02.3306^{\prime} \mathrm{N} \\
139^{\circ} 14.6292 \\
\end{array}$ & $\begin{array}{r}34^{\circ} 58.3834^{\prime} \mathrm{N} \\
139^{\circ} 12.9686^{\prime} \mathrm{E}\end{array}$ & SSW & $2160-2532$ & 6.15 \\
\hline KY0506H-08 & $\begin{array}{r}35^{\circ} 02.3286^{\prime} \mathrm{N} \\
139^{\circ} 14.7419^{\prime} \mathrm{E}\end{array}$ & $\begin{array}{r}34^{\circ} 58.2658^{\prime} \mathrm{N} \\
139^{\circ} 13.05666^{\prime} \mathrm{E}\end{array}$ & SSW & $442-827$ & 6.37 \\
\hline KY0506H-09 & $\begin{array}{r}35^{\circ} 01.9235^{\prime} \mathrm{N} \\
139^{\circ} 16.2376^{\prime} \mathrm{E}\end{array}$ & $\begin{array}{r}34^{\circ} 57.8544^{\prime} \mathrm{N} \\
139^{\circ} 14.5321^{\prime} \mathrm{E}\end{array}$ & SSW & $2021-2406$ & 6.37 \\
\hline KY0506H-10 & $\begin{array}{r}34^{\circ} 58.3517^{\prime} \mathrm{N} \\
139^{\circ} 13.3428^{\prime} \mathrm{E}\end{array}$ & $\begin{array}{r}35^{\circ} 02.4043, \mathrm{~N} \\
139^{\circ} 15.0572, \mathrm{E}\end{array}$ & NNE & $2-438$ & 8.04 \\
\hline KY0506H-11 & $\begin{array}{r}34^{\circ} 58.3009^{\prime} \mathrm{N} \\
139^{\circ} 13.5754^{\prime} \mathrm{E}\end{array}$ & $\begin{array}{r}35^{\circ} 02.3576^{\prime} \mathrm{N} \\
139^{\circ} 15.2722^{\prime} \mathrm{E}\end{array}$ & NNE & $831-1251$ & 6.95 \\
\hline KY0506H-12 & $\begin{array}{r}35^{\circ} 02.1644^{\prime} \mathrm{N} \\
139^{\circ} 15.45744^{\prime} \mathrm{E}\end{array}$ & $\begin{array}{r}34^{\circ} 58.0813^{\prime} \mathrm{N} \\
139^{\circ} 13.7305^{\prime} \mathrm{E}\end{array}$ & SSW & $1255-1629$ & 6.19 \\
\hline KY0506H-13 & $\begin{array}{r}34^{\circ} 58.1545^{\prime} \mathrm{N} \\
139^{\circ} 14.0709^{\prime} \mathrm{E}\end{array}$ & $\begin{array}{r}35^{\circ} 02.2138^{\prime} \mathrm{N} \\
139^{\circ} 15.78466^{\prime} \mathrm{E}\end{array}$ & NNE & $1633-2017$ & 6.35 \\
\hline KY0506H-A & $\begin{array}{r}35^{\circ} 01.2004^{\prime} \mathrm{N} \\
139^{\circ} 18.9594^{\prime} \mathrm{E}\end{array}$ & $\begin{array}{r}35^{\circ} 03.3092^{\prime} \mathrm{N} \\
139^{\circ} 11.3749^{\prime} \mathrm{E}\end{array}$ & WNW & $3503-4161$ & 10.87 \\
\hline KY0506H-B & $\begin{array}{r}35^{\circ} 03.0372{ }^{\prime} \mathrm{N} \\
139^{\circ} 11.4750 \\
\end{array}$ & $\begin{array}{r}35^{\circ} 00.9327^{\prime} \mathrm{N} \\
139^{\circ} 19.0499^{\prime} \mathrm{E}\end{array}$ & ESE & $2947-3499$ & 9.12 \\
\hline KY0506H-C & $\begin{array}{r}35^{\circ} 00.7888^{\prime} \mathrm{N} \\
139^{\circ} 18.8077^{\prime} \mathrm{E}\end{array}$ & $\begin{array}{r}35^{\circ} 02.9210^{\prime} \mathrm{N} \\
139^{\circ} 11.2140^{\prime} \mathrm{E}\end{array}$ & WNW & $2310-2943$ & 10.46 \\
\hline KY0506H-D & $\begin{array}{r}35^{\circ} 02.5494^{\prime} \mathrm{N} \\
139^{\circ} 11.7493^{\prime} \mathrm{E}\end{array}$ & $\begin{array}{r}35^{\circ} 00.5505^{\prime} \mathrm{N} \\
139^{\circ} 18.9190^{\prime} \mathrm{E}\end{array}$ & ESE & $1776-2306$ & 8.76 \\
\hline KY0506H-E & $\begin{array}{r}35^{\circ} 00.4223^{\prime} \mathrm{N} \\
139^{\circ} 18.7553^{\prime} \mathrm{E}\end{array}$ & $\begin{array}{r}35^{\circ} 02.4075^{\prime} \mathrm{N} \\
139^{\circ} 11.5511^{\prime} \mathrm{E}\end{array}$ & WNW & $1147-1772$ & 10.33 \\
\hline KY0506H-F & $\begin{array}{r}35^{\circ} 02.0846^{\prime} \mathrm{N} \\
139^{\circ} 11.8272^{\prime} \mathrm{E}\end{array}$ & $\begin{array}{r}35^{\circ} 00.1820^{\prime} \mathrm{N} \\
139^{\circ} 18.7272^{\prime} \mathrm{E} \\
\end{array}$ & ESE & $624-1143$ & 8.58 \\
\hline KY0506H-G & $\begin{array}{r}35^{\circ} 00.0424^{\prime} \mathrm{N} \\
139^{\circ} 18.5374^{\prime} \mathrm{E}\end{array}$ & $\begin{array}{r}35^{\circ} 01.9475^{\prime} \mathrm{N} \\
139^{\circ} 11.6917^{\prime} \mathrm{E}\end{array}$ & WNW & $2-620$ & 10.21 \\
\hline KY0506H-H & $\begin{array}{r}35^{\circ} 01.8240^{\prime} \mathrm{N} \\
139^{\circ} 11.3985^{\prime} \mathrm{E}\end{array}$ & $\begin{array}{r}34^{\circ} 59.8062^{\prime} \mathrm{N} \\
139^{\circ} 18.5729^{\prime} \mathrm{E}\end{array}$ & ESE & $2890-3407$ & 8.55 \\
\hline KY0506H-I & $\begin{array}{r}34^{\circ} 59.6422^{\prime} \mathrm{N} \\
139^{\circ} 18.4420^{\prime} \mathrm{E}\end{array}$ & $\begin{array}{r}35^{\circ} 01.6875^{\prime} \mathrm{N} \\
139^{\circ} 11.2051^{\prime} \mathrm{E}\end{array}$ & WNW & $2292-2886$ & 9.82 \\
\hline KY0506H-J & $\begin{array}{r}35^{\circ} 01.1616^{\prime} \mathrm{N} \\
139^{\circ} 12.2238^{\prime} \mathrm{E}\end{array}$ & $\begin{array}{r}34^{\circ} 59.4330^{\prime} \mathrm{N} \\
139^{\circ} 18.4158^{\prime} \mathrm{E} \\
\end{array}$ & ESE & $1825-2288$ & 7.66 \\
\hline KY0506H-K & $\begin{array}{r}34^{\circ} 59.2885^{\prime} \mathrm{N} \\
139^{\circ} 18.1780^{\prime} \mathrm{E} \\
\end{array}$ & $\begin{array}{r}35^{\circ} 01.1440^{\prime} \mathrm{N} \\
139^{\circ} 11.5856^{\prime} \mathrm{E} \\
\end{array}$ & WNW & $1239-1821$ & 9.62 \\
\hline KY0506H-L & $\begin{array}{r}35^{\circ} 01.1731^{\prime} \mathrm{N} \\
139^{\circ} 10.6699^{\prime} \mathrm{E} \\
\end{array}$ & $\begin{array}{r}34^{\circ} 59.0420^{\prime} \mathrm{N} \\
139^{\circ} 18.2670^{\prime} \mathrm{E}\end{array}$ & ESE & $2579-3153$ & 9.49 \\
\hline KY0506H-M & $\begin{array}{r}35^{\circ} 01.0743^{\prime} \mathrm{N} \\
139^{\circ} 10.6367^{\prime} \mathrm{E}\end{array}$ & $\begin{array}{r}34^{\circ} 58.9265^{\prime} \mathrm{N} \\
139^{\circ} 18.3530^{\prime} \mathrm{E}\end{array}$ & ESE & $654-1235$ & 9.60 \\
\hline KY0506H-N & $\begin{array}{r}34^{\circ} 58.9059^{\prime} \mathrm{N} \\
139^{\circ} 18.0740^{\prime} \mathrm{E} \\
\end{array}$ & $\begin{array}{r}35^{\circ} 01.0302^{\prime} \mathrm{N} \\
139^{\circ} 10.4532^{\prime} \mathrm{E}\end{array}$ & WNW & $1951-2574$ & 10.28 \\
\hline KY0506H-O & $\begin{array}{r}34^{\circ} 58.8110^{\prime} \mathrm{N} \\
139^{\circ} 17.9767^{\prime} \mathrm{E} \\
\end{array}$ & $\begin{array}{r}35^{\circ} 00.9519^{\prime} \mathrm{N} \\
139^{\circ} 10.3981^{\prime} \mathrm{E}\end{array}$ & WNW & $2-650$ & 10.71 \\
\hline KY0506H-P & $\begin{array}{r}35^{\circ} 00.8007^{\prime} \mathrm{N} \\
139^{\circ} 10.5002^{\prime} \mathrm{E}\end{array}$ & $\begin{array}{r}34^{\circ} 58.6529^{\prime} \mathrm{N} \\
139^{\circ} 18.1459^{\prime} \mathrm{E}\end{array}$ & ESE & $1405-1946$ & 8.94 \\
\hline KY0506H-Q & $\begin{array}{r}34^{\circ} 58.9571^{\prime} \mathrm{N} \\
139^{\circ} 16.3319^{\prime} \mathrm{E}\end{array}$ & $\begin{array}{r}35^{\circ} 00.6646^{\prime} \mathrm{N} \\
139^{\circ} 10.2618^{\prime} \mathrm{E}\end{array}$ & WNW & $912-1401$ & 8.09 \\
\hline KY0506H-R & $\begin{array}{r}35^{\circ} 00.4153^{\prime} \mathrm{N} \\
139^{\circ} 10.3483^{\prime} \mathrm{E}\end{array}$ & $\begin{array}{r}34^{\circ} 58.7716^{\prime} \mathrm{N} \\
139^{\circ} 16.2264^{\prime} \mathrm{E}\end{array}$ & ESE & $488-908$ & 6.95 \\
\hline KY0506H-S & $\begin{array}{r}34^{\circ} 58.6444^{\prime} \mathrm{N} \\
139^{\circ} 15.9770^{\prime} \mathrm{E}\end{array}$ & $\begin{array}{r}35^{\circ} 00.2936^{\prime} \mathrm{N} \\
139^{\circ} 10.1255^{\prime} \mathrm{E}\end{array}$ & WNW & $2-484$ & 7.97 \\
\hline KY0506H-T & $\begin{array}{r}35^{\circ} 00.0455^{\prime} \mathrm{N} \\
139^{\circ} 10.2017^{\prime} \mathrm{E}\end{array}$ & $\begin{array}{r}34^{\circ} 58.3893^{\prime} \mathrm{N} \\
139^{\circ} 16.0797^{\prime} \mathrm{E}\end{array}$ & ESE & $1897-2309$ & 6.81 \\
\hline KY0506H-U & $\begin{array}{r}34^{\circ} 58.2552^{\prime} \mathrm{N} \\
139^{\circ} 15.7926^{\prime} \mathrm{E}\end{array}$ & $\begin{array}{r}34^{\circ} 59.9069^{\prime} \mathrm{N} \\
139^{\circ} 09.9530^{\prime} \mathrm{E}\end{array}$ & WNW & $1404-1893$ & 8.09 \\
\hline KY0506H-V & $\begin{array}{r}34^{\circ} 59.6508^{\prime} \mathrm{N} \\
139^{\circ} 10.0595^{\prime} \mathrm{E}\end{array}$ & $\begin{array}{r}34^{\circ} 58.0137^{\prime} \mathrm{N} \\
139^{\circ} 15.9187^{\prime} \mathrm{E}\end{array}$ & ESE & $980-1400$ & 6.95 \\
\hline KY0506H-W & $\begin{array}{r}34^{\circ} 57.8770^{\prime} \mathrm{N} \\
139^{\circ} 15.6841^{\prime} \mathrm{E}\end{array}$ & $\begin{array}{r}34^{\circ} 59.5244^{\prime} \mathrm{N} \\
139^{\circ} 09.8091^{\prime} \mathrm{E}\end{array}$ & WNW & $471-976$ & 8.35 \\
\hline KY0506H-X & $\begin{array}{r}34^{\circ} 59.2920^{\prime} \mathrm{N} \\
139^{\circ} 09.8692^{\prime} \mathrm{E}\end{array}$ & $\begin{array}{r}34^{\circ} 57.6365^{\prime} \mathrm{N} \\
139^{\circ} 15.7533^{\prime} \mathrm{E}\end{array}$ & ESE & $2-467$ & 7.69 \\
\hline & & & & Total & 299.91 \\
\hline
\end{tabular}

Line lengths in this table were derived from the calculation of number of shots multiplied by $16.5 \mathrm{~m}$ ( 8 seconds pop interval approximately corresponds $16.5 \mathrm{~m}$ when 4 knots cruising speed). 
Preamp: $39 \mathrm{~dB}$

The towing depth of SIG16 streamer was variable from $3.1 \mathrm{~m}$ to $6.7 \mathrm{~m}$ below sea surface.

\section{Recording system}

The Triton Erics' Delph Seismic system was used for data recording. The main feature of the system and parameters are as follows:

Type of system: Delph Seismic

Monitor: EPC The model GSP-1086 Printer

Recording format: SEG-Y

Recording length: 7 seconds

Sampling rate: $1 \mathrm{~ms}$

\subsection{Cluster gun system}

The cluster gun system is effective to obtain images for the shallower part, down to a few hundred meters. Fig. 4 shows the photograph of the system onboard KAIYO.

Specifications:
(1) Manufacturer:
Bolt Technology
(2) Type of airgun: 2800LL-X Cluster
(3) Volume:
20 cuin $\times 2$
(4) Air pressure:
2000 psi
(5) Source Depth:
$1.3 \mathrm{~m}$
(6) Peak-peak:
7 bar-m
(7) Primary to bubble:
43.8
(8) Dominant frequency $(-6 \mathrm{~dB})$ :
$50 \mathrm{~Hz}-530 \mathrm{~Hz}$
(9) Depth sensor:
N/A
(10) Gun Controller:
GCS90

\subsection{Data Processing}

SCS reflection profiles are processed for the purpose of quality control. The data processing for the overall Sagami Bay profiles contains noisy-trace editing, 14$180 \mathrm{~Hz}$ band-pass filtering and time migration $(\mathrm{Vp}=1500 \mathrm{~m} / \mathrm{s})$. The data processing of Hatsushima site profiles contains noisy-trace editing, 14-180 Hz (GI gun), 45-500 Hz (Cluster gun) band-pass filtering and

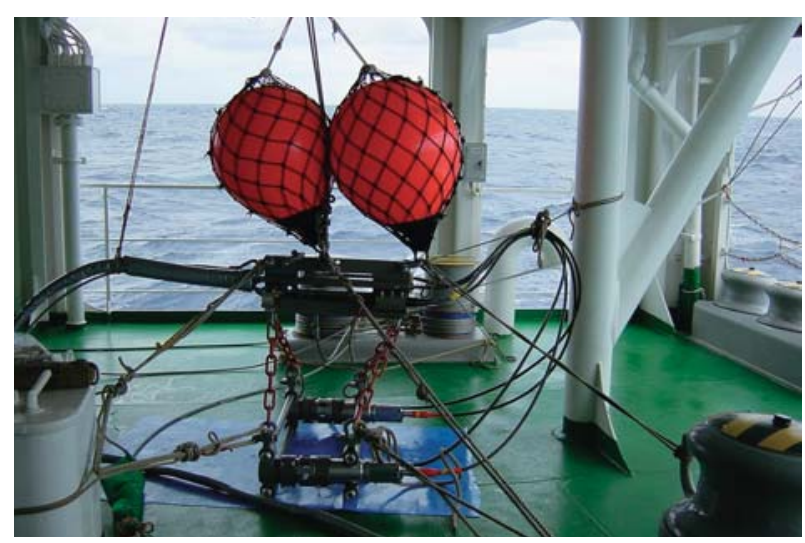

Figure 4: Photograph of the cluster gun system ready for launching onboard KAIYO. time migration $(\mathrm{Vp}=1500 \mathrm{~m} / \mathrm{s})$.

\section{Outline of obtained data}

Approx. $852 \mathrm{~km}$ of SCS data was recorded for the overall Sagami Bay survey. And it was particularly mentioned that 5 survey lines (total length of approx. 79 $\mathrm{km}$ ) crossing ship lane for Tokyo bay were recorded successfully during midnights of so called "golden week". The total ship time for this survey was 9 days \& 20 hours, and SCS survey time was 4 days \& 19 hours (49\% of the total ship time). The attached SCS images (Fig. 5) were displayed through FOCUS software, but $120 \mathrm{~ms}$ system delay and datum correction were not yet applied.

For the Hatsushima intensive survey, we could obtain very clear images down to $1 \mathrm{sec}$. (TWT). An intrusion to the east of Izu Peninsula can be traced for the most of $\mathrm{E}-\mathrm{W}$ lines (C through $\mathrm{U}$ ). This is also clearly recognized at a crossing line 9. A diaper-like structure, with its diameter $\sim 500 \mathrm{~m}$, was identified at lines 9 and $\mathrm{U}$.

The profiles shown in this report is preliminary. We need to correct for the position of the streamer first. Final interpretation will be reported afterwards.

\section{Acknowledgments}

We thank Captain Hitoshi Tanaka, crew and technical staffs of R/V KAIYO, for their kind and thoughtful support during SCS survey. This survey was achieved under IFREE, CDEX and MARITEC of JAMSTEC.

\section{References}

1) Iwabuchi, Y., Kato, Y., Hashimoto, F., Kondo, T. and Shinbayashi, K., Multi-channel seismic reflection survey of the Sagami Bay. J. Jpn. Soc. Mar. Surv. Tech. 3, 39-51, 1990 (in Japanese).

2) Kato, S., Submarine topography and geological structure of Sagami Bay. The Quaternary Research 38, 469-477, 1999.

3) Kato, S., Y. Iwabuchi, A. Asada, Y. Kato, S. Kikuchi, S. Kokuta, K. Kusunoki, and K. Watanabe, Crustal structure and tectonic landform of Sagami Bay, J. Geography, 102, 399-406, 2003 (in Japanese).

4) Okada, Y., E. Yamamoto, and T. Ohkubo, Coswarm and preswarm crustal deformation in the eastern Izu Peninsula, central Japan, J. Geophys. Res., 105, 681-692, 2000.

5) Okino, K., Nishizawa, A. and Asada, A., Crustal structure survey in the northwestern part of the Sagami Trough. Rep. Hydrographic Res. 30, 383-393, 1994 (in Japanese with English abstract). 

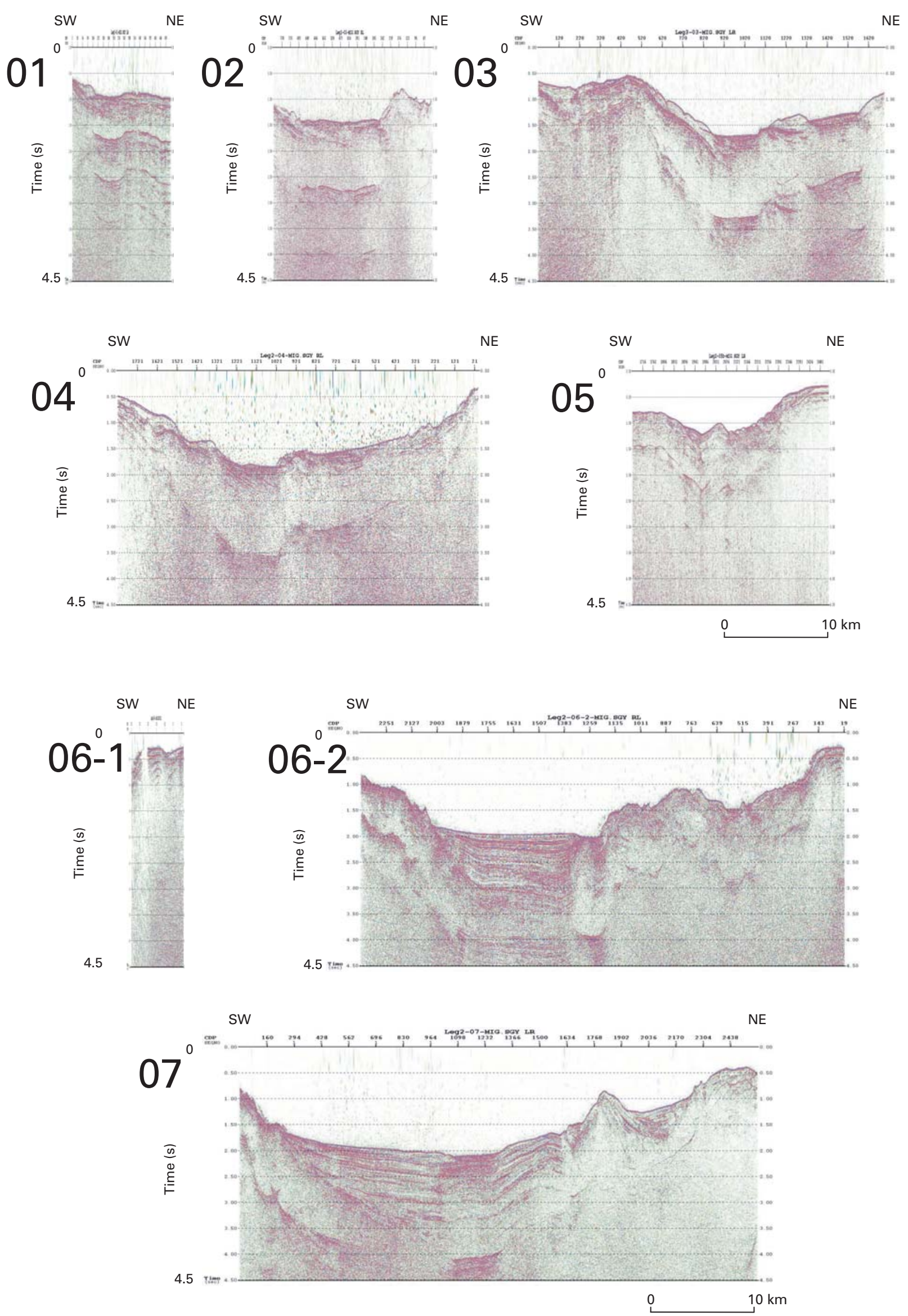

Figure 5: SCS profiles in the overall Sagami Bay area. Vertical axis is a two-way travel time in second. Horizontal axis is the shot point (SP) number, with relative distance scale attached. 

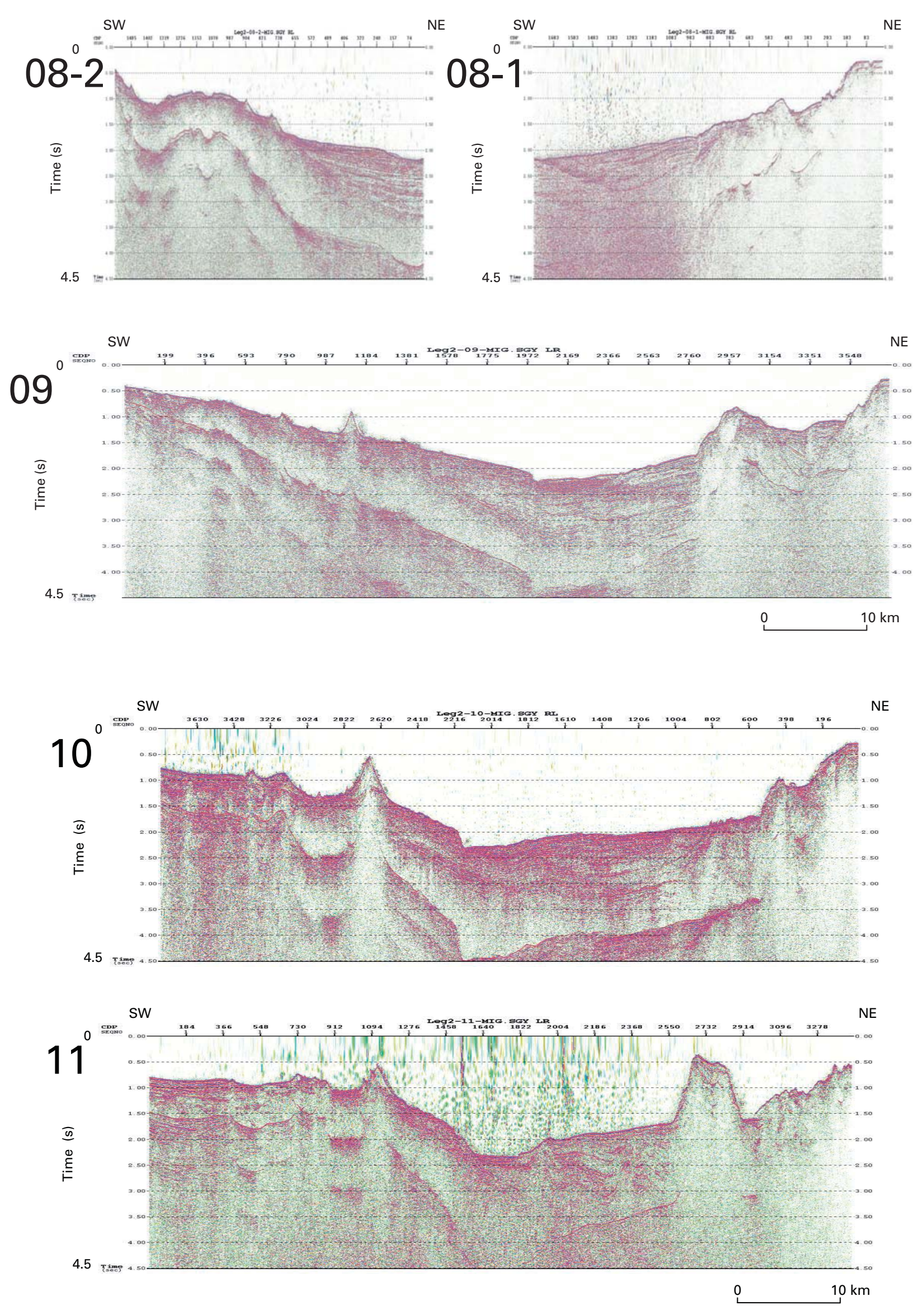

Figure 5: (Continued) 

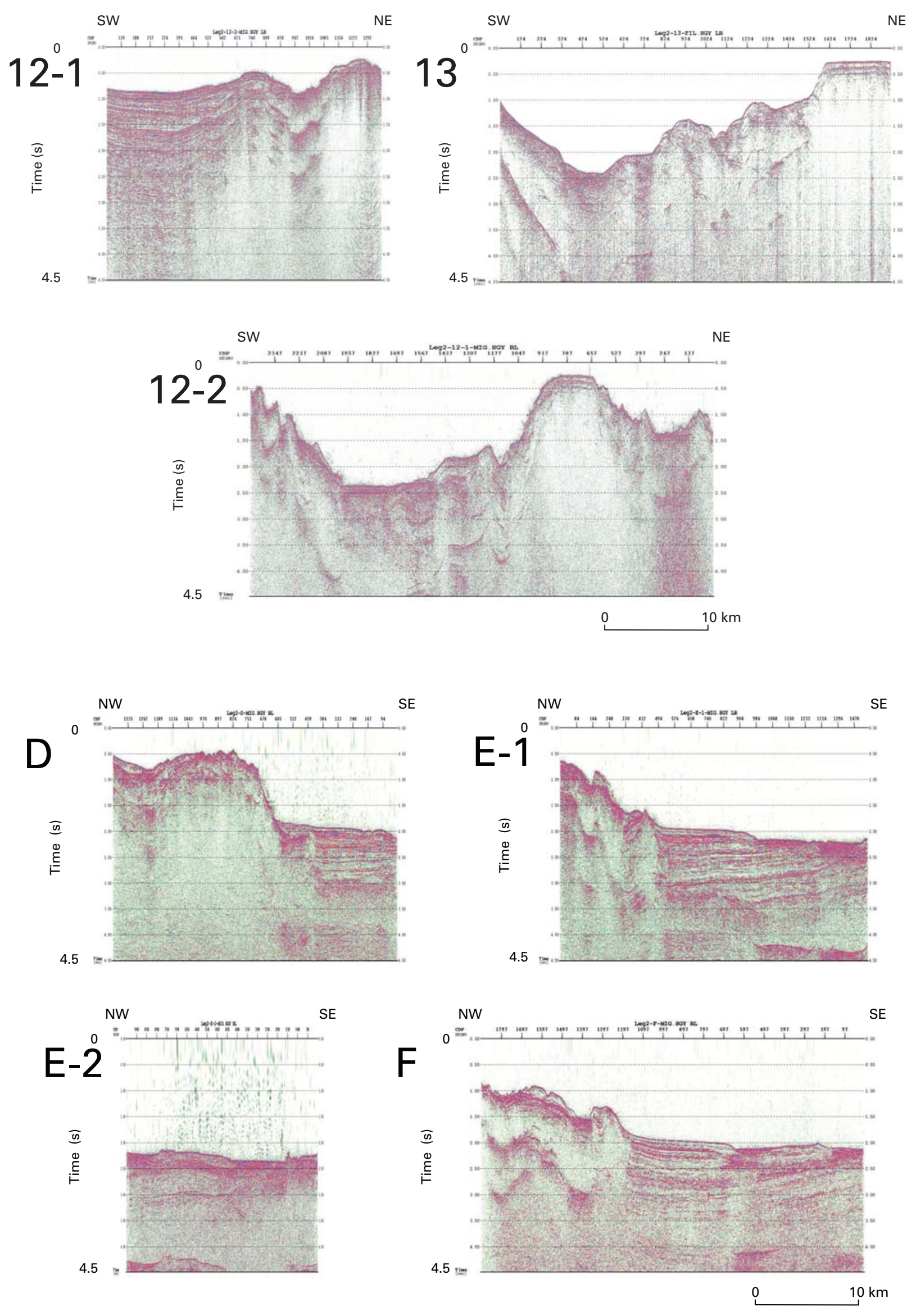

Figure 5: (Continued) 

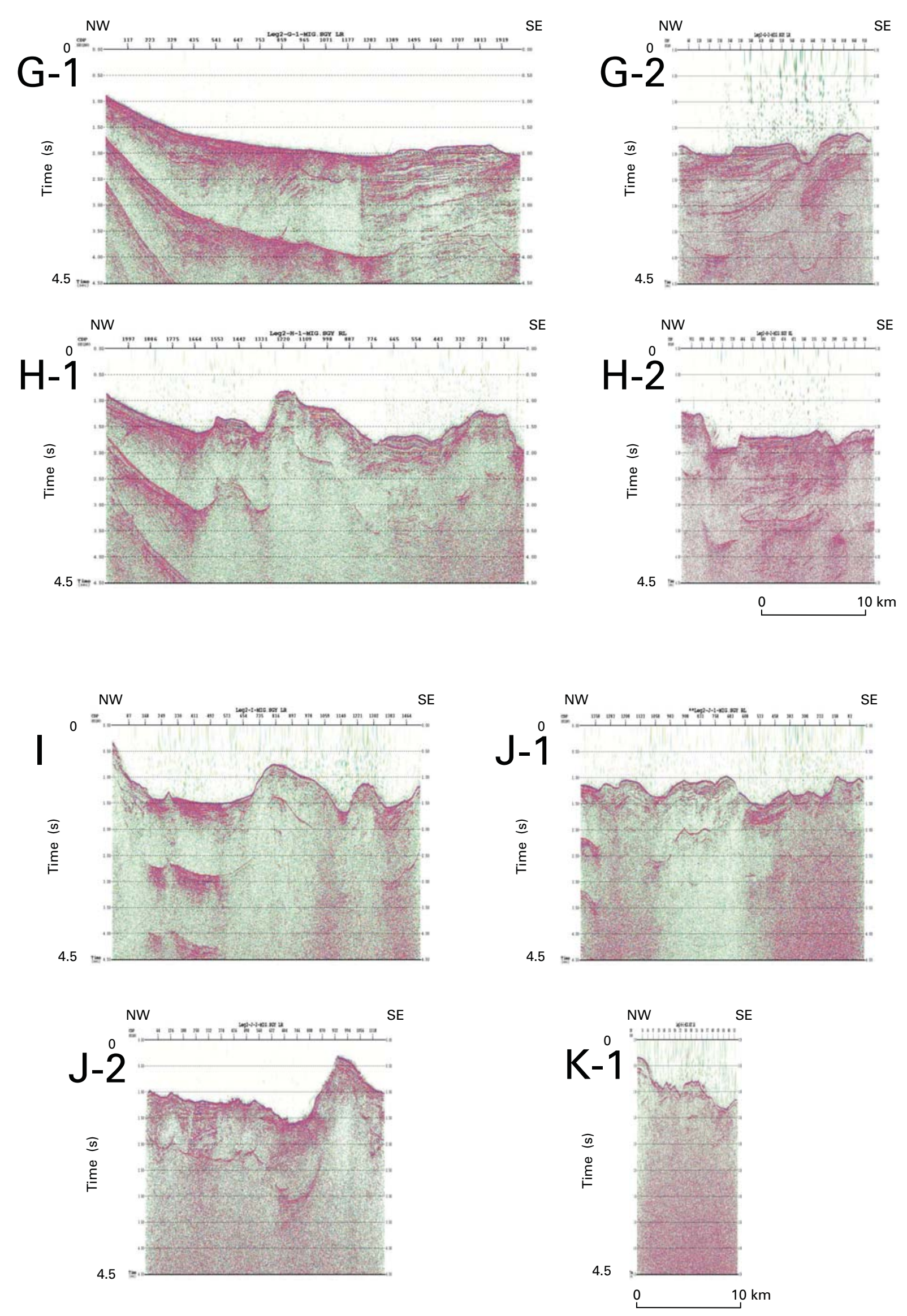

Figure 5: (Continued) 


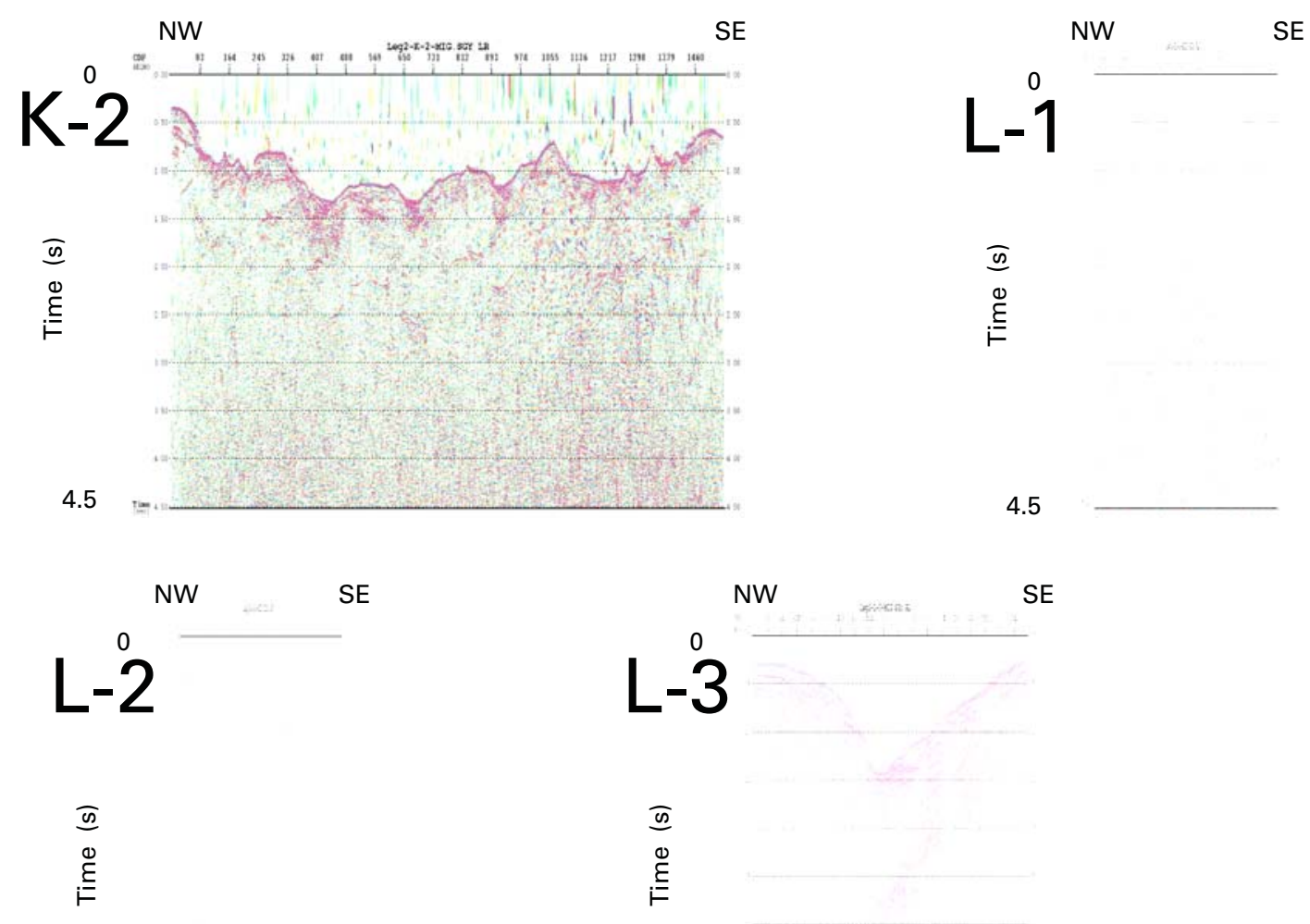

4.5 4.5

Figure 5: (Continued) 

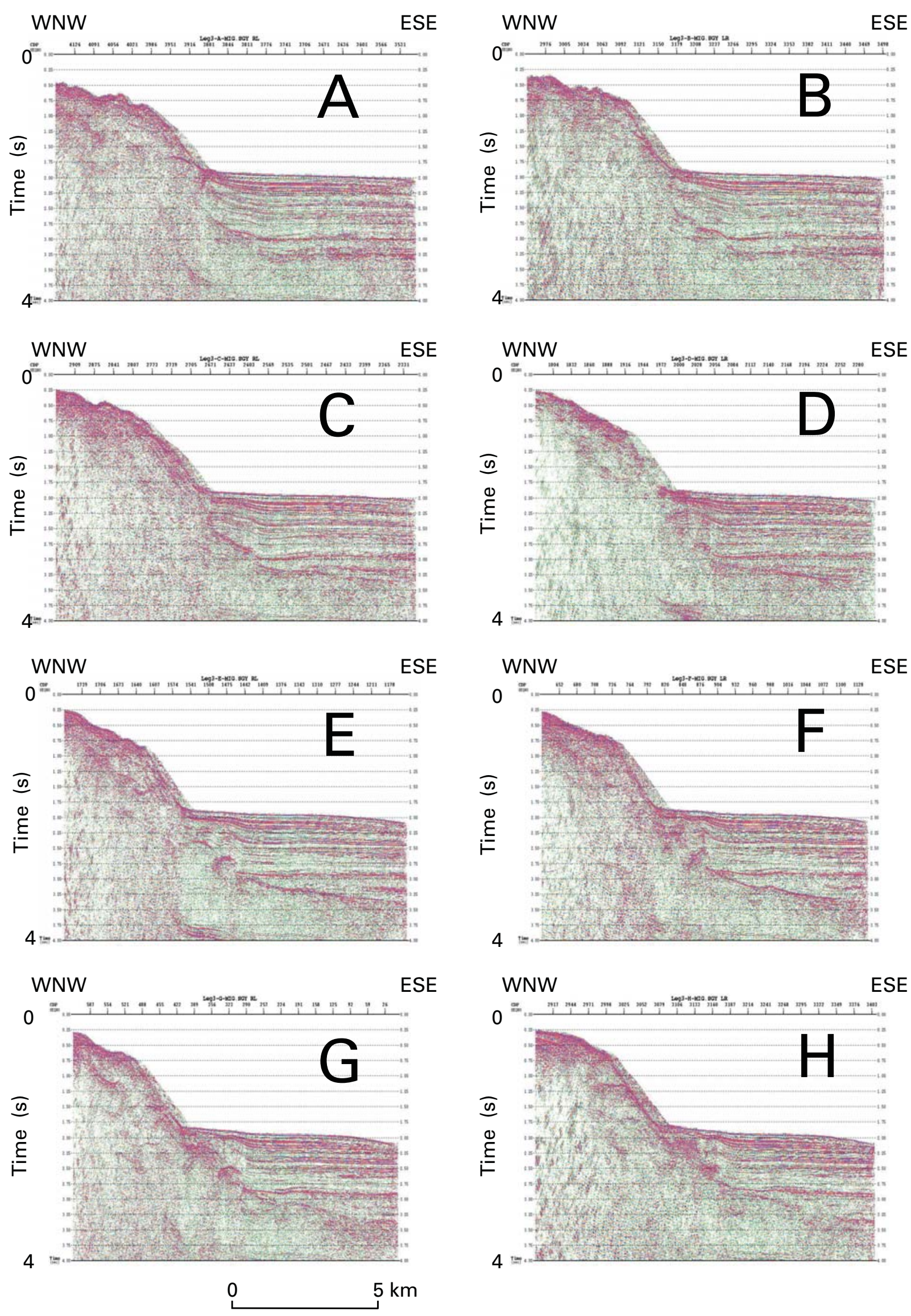

Figure 6: Seismic reflection profiles of line A-Z (Hatsushima site) by using GI gun in the WNW-ESE direction. Vertical axis is a two-way travel time in second. Horizontal axis is the SP number, with relative distance scale attached. 

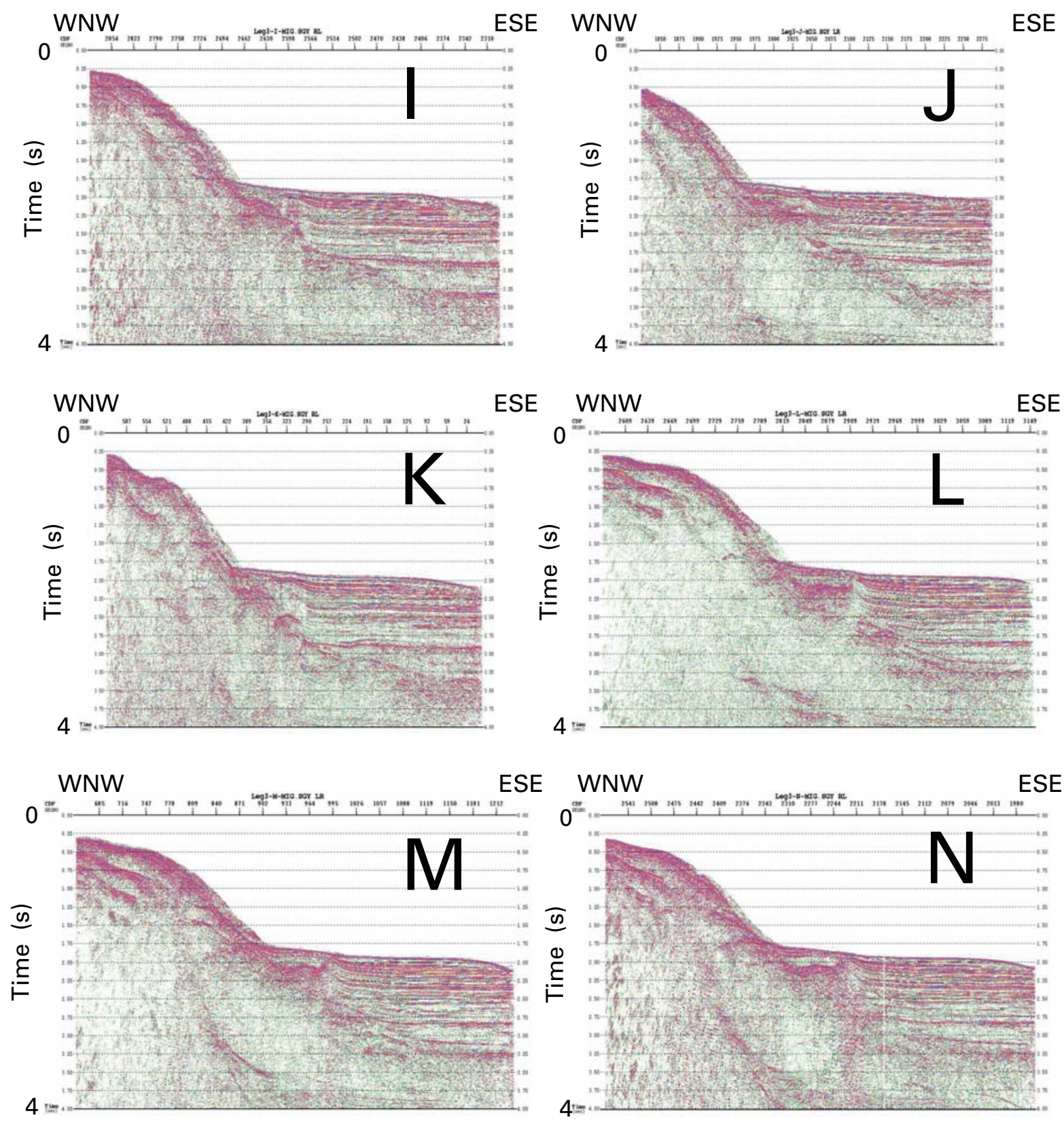

ESE WNW

ESE
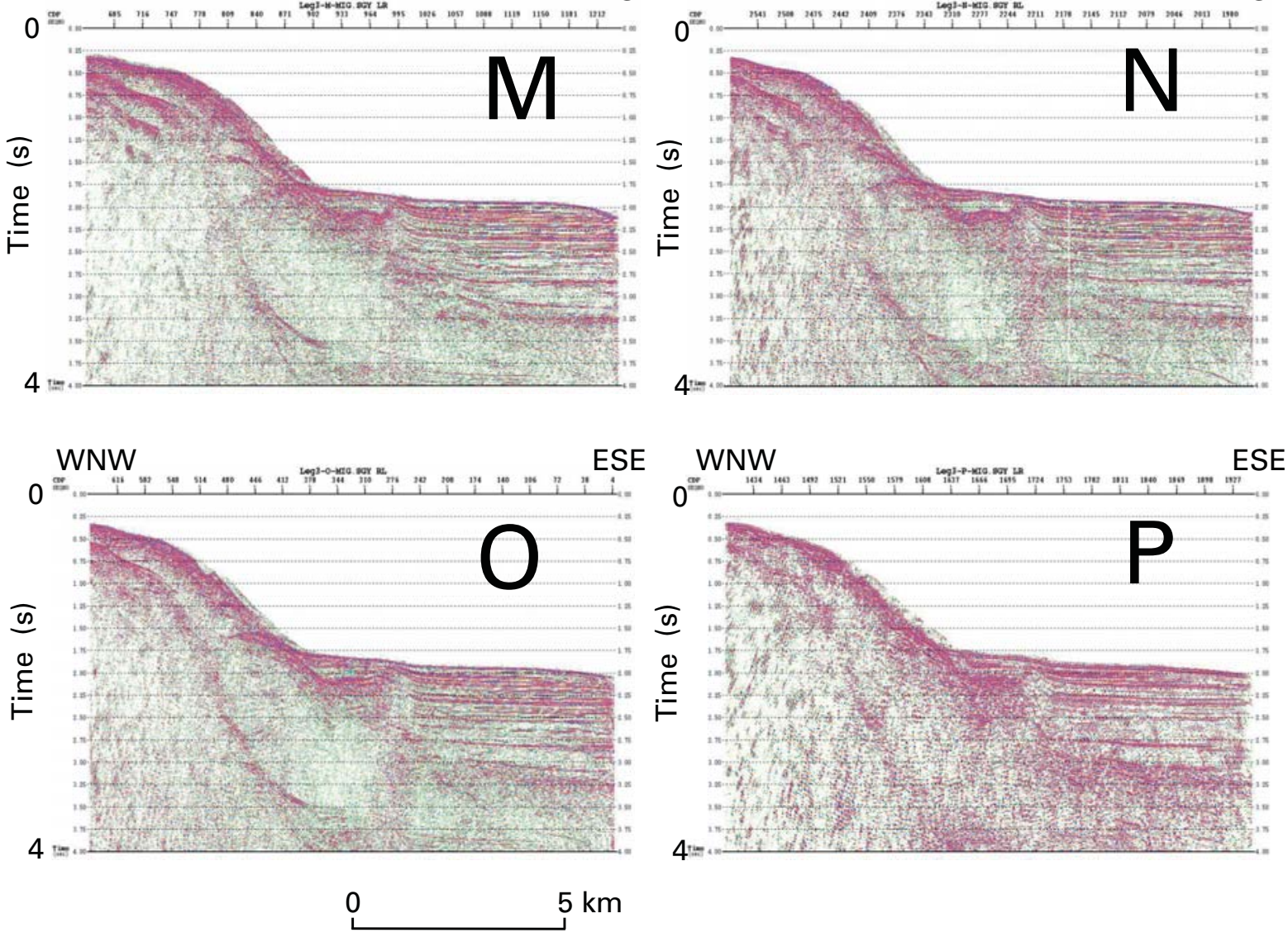

Figure 6: (Continued) 

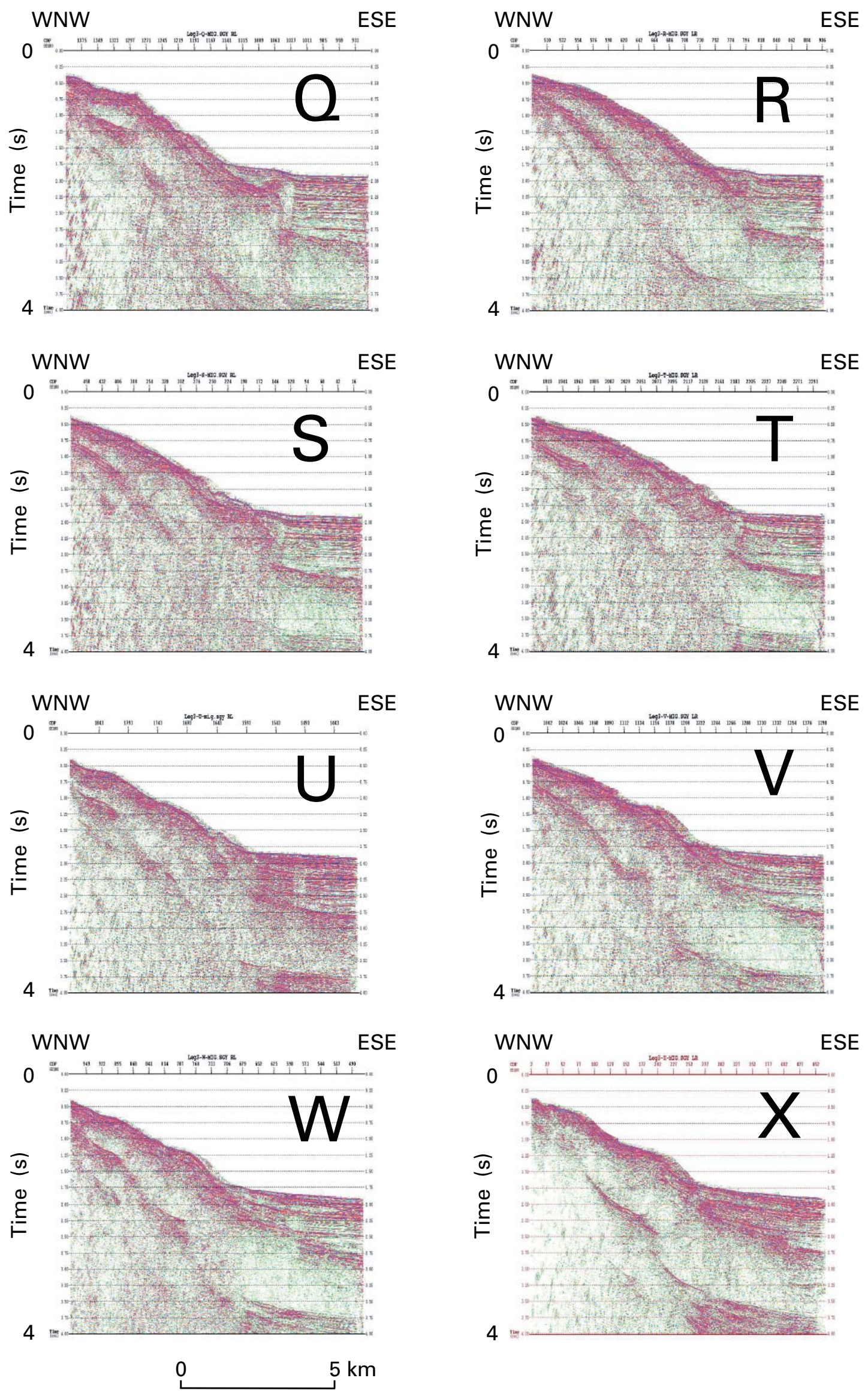

Figure 6: (Continued) 

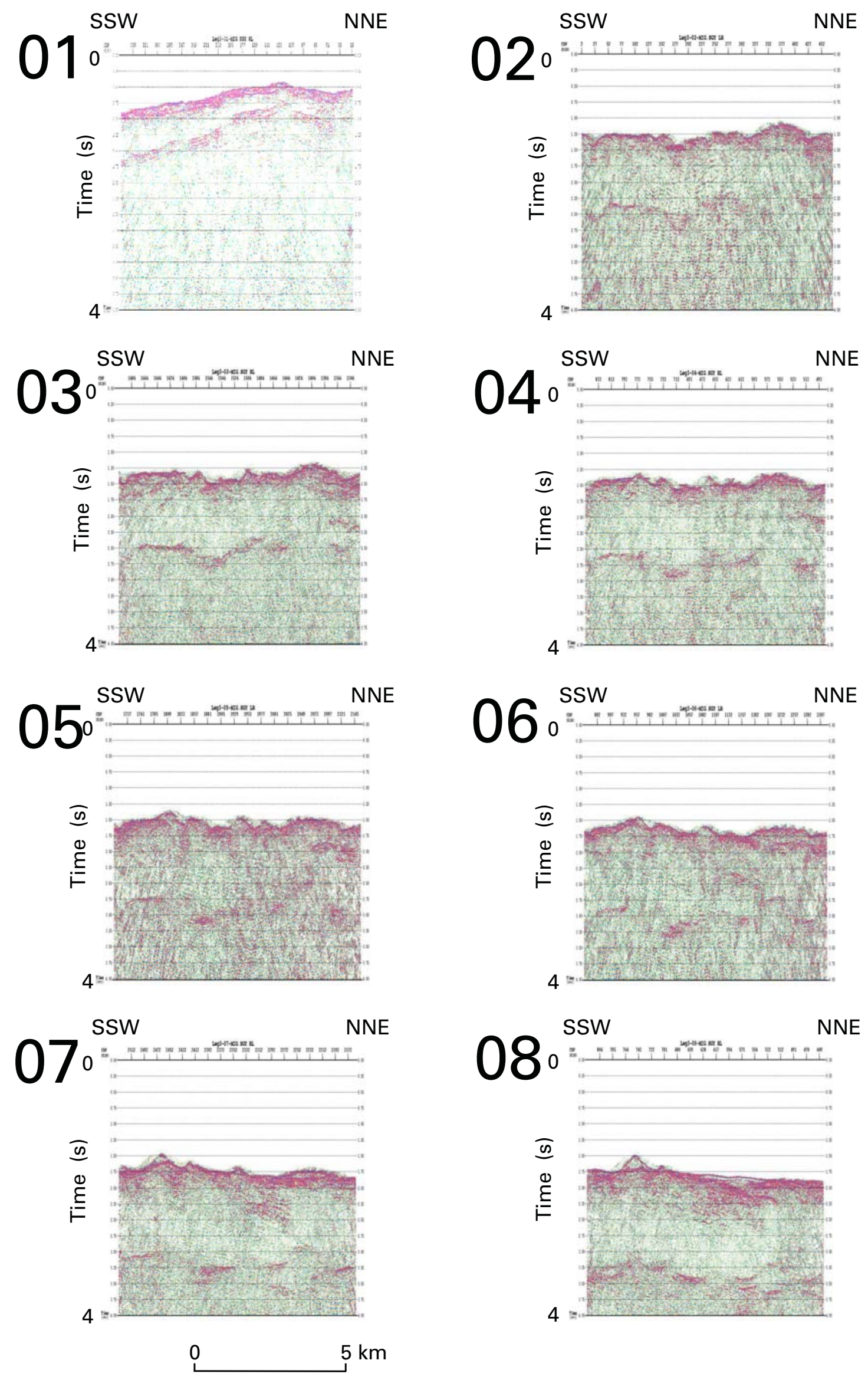

Figure 7: Seismic reflection profiles of line 01-13 (Hatsushima site) by using GI gun in the NNE-SSW direction. Vertical axis is a two-way travel time in second. Horizontal axis is the SP number, with relative distance scale attached. 

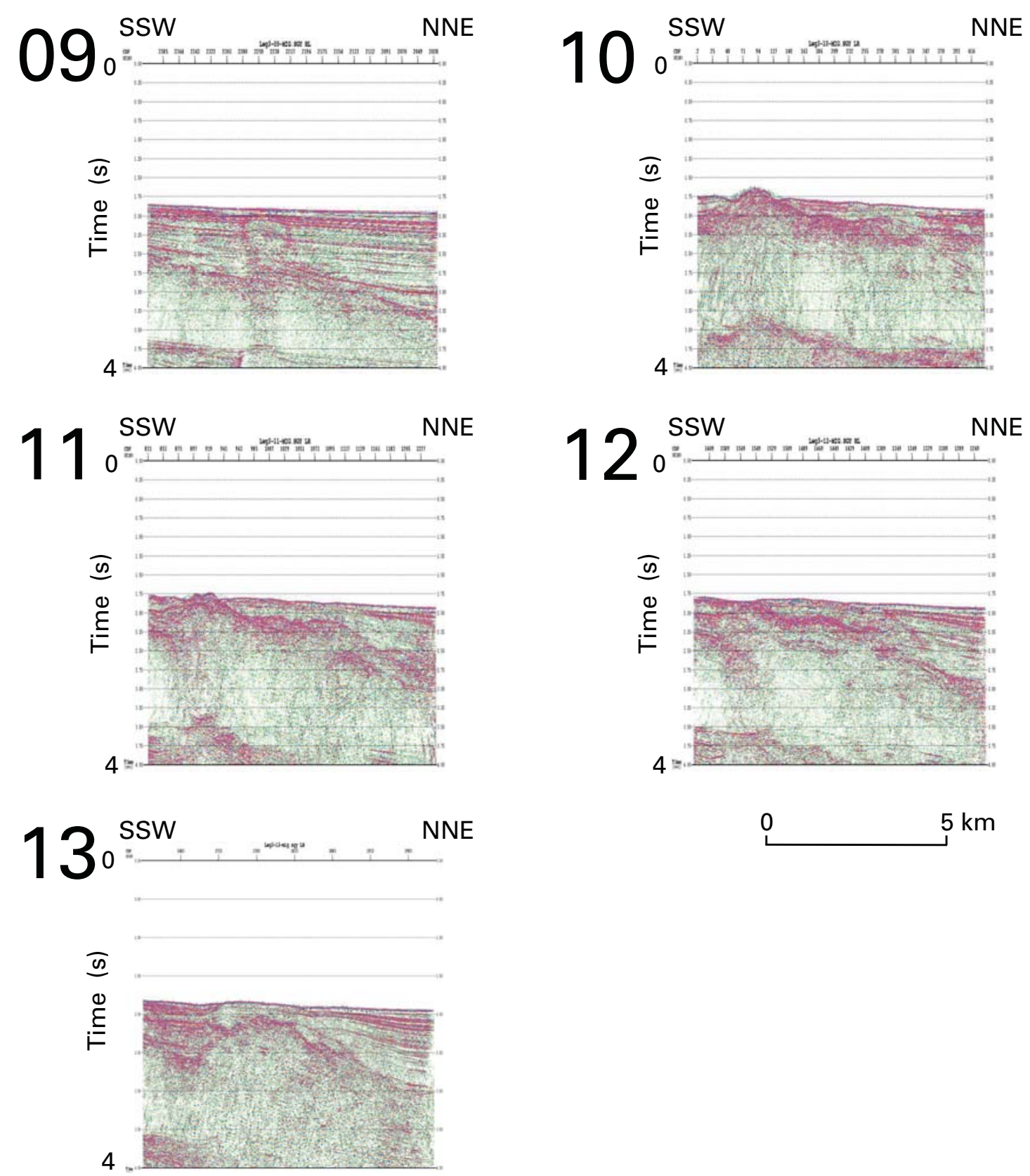

Figure 7: (Continued) 


\section{PM1c}

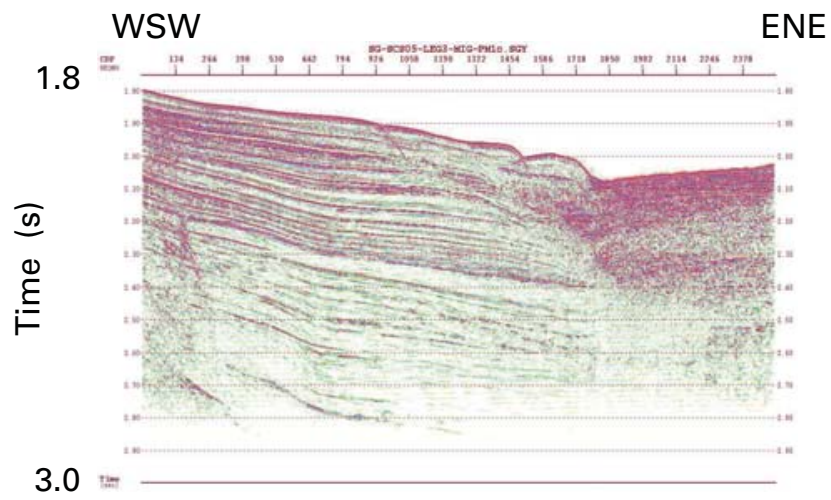

PM2c

SSE NNW

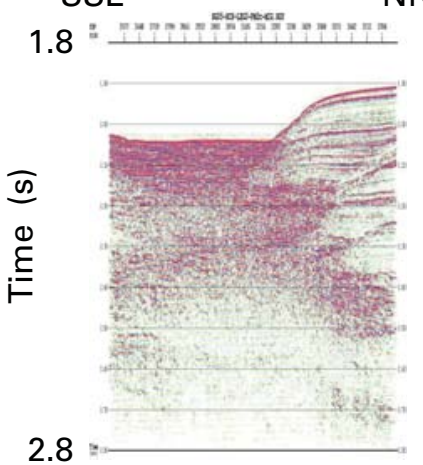

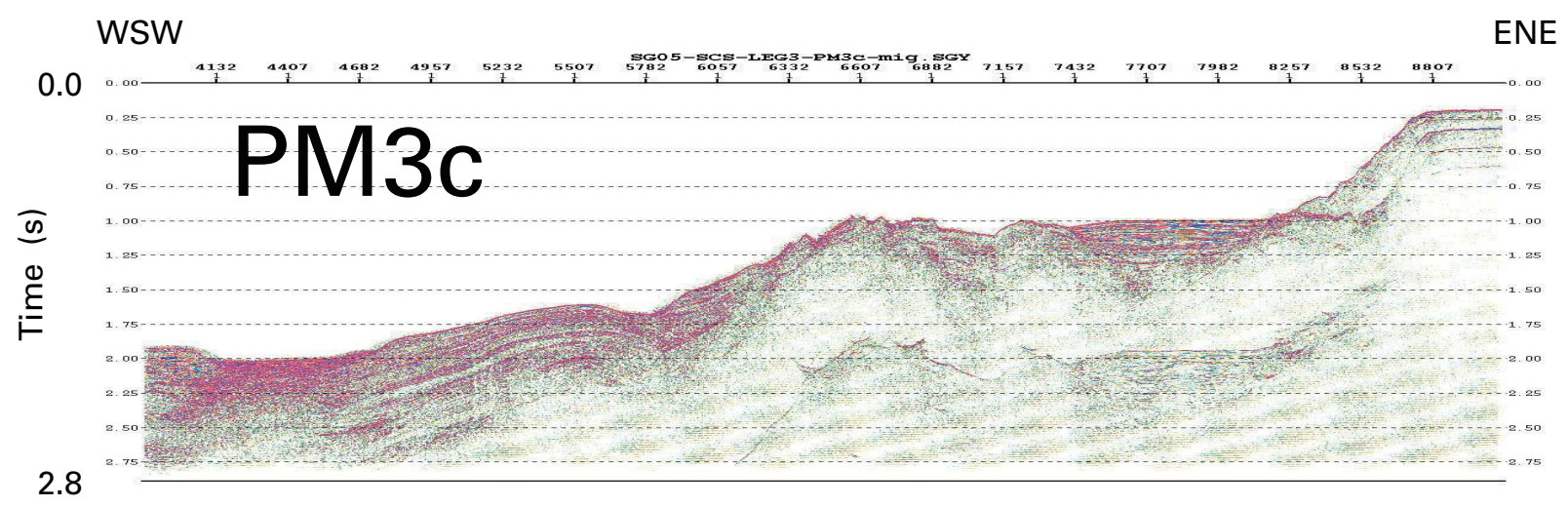

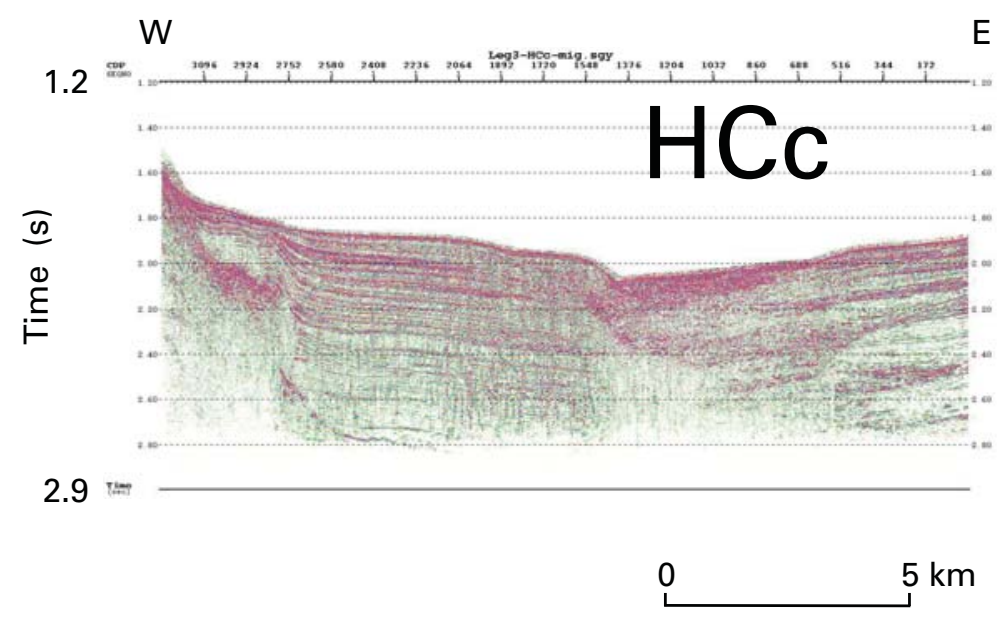

Figure 8: Seismic reflection profiles obtained using Cluster gun. The vertical axis is Two way travel time in sec. Vertical axis is a two-way travel time in second. Horizontal axis is the SP number, with relative distance scale attached. Note that the scale of vertical axis is different for each profile. 